\title{
The effect of sevoflurane anesthesia for dental procedure on neurodevelopment in children: a prospective, equivalence, controlled trial
}

\section{Pinping Zhou}

Chongqing Medical University Stomatology College

Chao Zhang

Chongqing Medical University Stomatology College

Guijin Huang

Chongqing Medical University Stomatology College

Yuan $\mathrm{Hu}$

Chongqing Medical University Stomatology College

Wenzhu Ma

Chongqing Medical University Stomatology College

Cong Yu (D 500158@hospital.cqmu.edu.cn )

Chongqing Medical University https://orcid.org/0000-0002-1957-574X

\section{Research article}

Keywords: neurodevelopment, sevoflurane, general anesthesia, dental procedure, children

Posted Date: August 10th, 2020

DOl: https://doi.org/10.21203/rs.3.rs-33454/v2

License: () (1) This work is licensed under a Creative Commons Attribution 4.0 International License. Read Full License 


\section{Abstract}

Background Dental procedures under general anesthesia (DGA) was found to improve the oral healthrelated quality of children's life. However, some parents and pediatricians expressed concern about the neurotoxicity of general anesthesia. The purpose of this trial was to investigate whether DGA in children has an adverse effect on neurodevelopment. Methods In this prospective, assessor-masked, controlled, equivalence trial, we recruited 340 children younger than 7 years who were undergoing caries treatment between Feb 1, 2019, and Aug 31, 2019, without factors affecting neurodevelopment. They received either sevoflurane-based general anesthesia or awake-local anesthesia. The Wechsler Preschool and Primary Scale of Intelligence-Fourth Edition Chinese version was used to evaluate the neurodevelopment of children at six months after surgery, and the Full-Scale IQ (FSIQ) was selected as the primary outcome. Predefine the $95 \% \mathrm{Cl}$ of a difference in means within five (1/3 SD) as the equivalence margin. Results The outcome data were obtained from 129 children in the general anesthesia group and 144 in the local anesthesia group. The median length of general anesthesia was $130 \mathrm{~min}$ (IQR 110 - 160). There was equivalence in means of FSIQ score between the general anesthesia group and the local anesthesia group (local minus general anesthesia $0.46,95 \% \mathrm{Cl}-2.35$ to 1.61 ). There was no significant difference in FSIQ scores between different age groups and different anesthesia durations. Just the mother's education could affect the primary outcome. Conclusions In this trial, prolonged DGA with a sevofluraneonly anesthetic in preschool children, does not adversely affect neurodevelopment at six months after surgery compared with awake-local anesthesia.

\section{Background}

Recent studies have found that the prevalence of caries in children aged 3 to 5 years was $50.8 \%$ to $71.9 \%$ in China ${ }^{1}$. A growing number of children have caries of multiple teeth, and the treatment time is longer, usually more than $1 \mathrm{~h}$. For children with severe dental anxiety, dental procedures under general anesthesia (DGA) is common practice for anesthesia for caries treatment. DGA has been demonstrated to have a higher success rate and safety ${ }^{2,3}$. In our previous research, we found that DGA with sevoflurane improved the oral health-related quality of children's life, and most families expressed high satisfaction ${ }^{4}$.

Since general anesthesia was found to be neurotoxic in animal models ${ }^{5-9}$, many parents and pediatricians expressed concern and hesitated whether to delay the procedure. However, several clinical studies did not find that general anesthesia harmed the development of children, including inguinal herniorrhaphy, orthopedic surgery, and gastrointestinal surgery ${ }^{10-15}$. They had a shorter duration of surgery or used multiple anesthetics, and there was no relevant research in the field of dental procedure.

Therefore, the purpose of our trial was to investigate the effect of prolonged exposure to sevoflurane for DGA on the neurodevelopment in healthy preschool children.

\section{Methods}




\section{Study Design}

A prospective, assessor-blind, controlled, equivalence trial was performed to compare neurodevelopmental outcomes assessed by the Wechsler Preschool and Primary Scale of IntelligenceFourth Edition (WPPSI-IV) Chinese version (CN) at six months after surgery for preschool children undergoing caries treatment under sevoflurane-based general anesthesia or awake-local anesthesia. The WPPSI-IV (CN) is a test scale used to assess general intellectual function and neurocognitive development. It is mainly used in clinical and research fields, and it has a high-quality norm in China and high clinical validity. There are extensive academic literature and application experience about the WPPSIIV (CN). The WPPSI-IV (CN) includes thirteen subtests, and the composite score includes the Full-Scale IQ (FSIQ), main index, and auxiliary index. We selected the FSIQ and main indexes, including Verbal Comprehension Index (VCI), Visual-Spatial Index (VSI), Fluid Reasoning Index (FRI), Working Memory Index (WMI), and Processing Speed Index (PSI), from the composite score of WPPSIIV (CN) as the evaluation indicators. The primary outcome for the analysis on a per-protocol basis was prespecified to be the FSIQ, and the sample size was calculated based on the FSIQ score. The mean of the FSIQ score in the reference population is 100 , and the standard deviation is 15 . Assuming a sample ratio of $1: 1$, a significance level of 0.05 , and a test power of $80 \%$, the trial would need 308 patients. Enrolling roughly 340 patients would allow for $10 \%$ with dropping out. The secondary outcomes for this analysis were selected $\mathrm{VCI}, \mathrm{VSI}, \mathrm{FRI}, \mathrm{WMI}$, and $\mathrm{PSI}$, which are mainly used to evaluate the ability of knowledge expression and spoken language, visual-spatial information processing and vision-action coordination, fluid intelligence and inductive reasoning, visual working memory and attention, and cognitive flexibility and visual scan.

From Feb 1, 2019, to Aug 31, 2019, 170 children receiving general anesthesia and 170 children receiving local anesthesia were observed in the Stomatological Hospital of Chongqing Medical University, and the written consent obtained from parents or guardians. The anesthetists, pediatricians, and parents were aware of group allocation, but the researchers who administered the neurofunctional assessments were not. The study had been treated according to the declaration of Helsinki. This trial was registered with the Chinese Clinical Trial Registry (number ChiCTR1800015216), and the ethics committee approval of the Stomatological Hospital of Chongqing Medical University was obtained (number CQHS-IRB-2018-01).

\section{Participants}

Inclusion criteria included healthy children less than 7 years old scheduled for caries treatment, more than 6 teeth treated, and expected duration of surgery more than $1 \mathrm{~h}$. Exclusion criteria were any contraindication for anesthesia, previous exposure to general anesthesia, moderate to severe preterm infants (gestational age no more than 33 weeks), very low birth weight infant (birth weight less than $1500 \mathrm{~g}$ ), any known neurological injury or developmental issues, other known diseases that might affect neurodevelopment, epileptic, deaf-mute or blind, and any reason that might make follow-up difficult. Eligible children were recruited in the Department of Pediatric Dentistry or Anesthesiology of the 
Stomatological Hospital of Chongqing Medical University. During the research, children were excluded if they suffered brain injuries, underwent any other surgery, or failed to complete all procedures as protocol.

\section{Anesthesia}

The awake-local anesthesia group received the local infiltration anesthesia after the behavior induction. Children under 4 years old were given $2 \%$ lidocaine hydrochloride injection ( $5 \mathrm{ml}: 0.1 \mathrm{~g}$, Southwest Pharmaceutical Co.; SFDA no.H50020038), with the total dose no more than $4 \mathrm{mg} / \mathrm{Kg}$. Children aged 4 years and older were given $4 \%$ articaine hydrochloride and epinephrine tartrate injection (1.7ml: $68 \mathrm{mg}$, Produits Dentaires Pierre Rolland; SFDA no.H20140732), with the maximum dosage not exceeding 5 $\mathrm{mg} / \mathrm{Kg}$.

The general anesthesia group only received sevoflurane $(120 \mathrm{ml}$, Shanghai Hengrui Pharmaceutical Co.; SFDA no.H20070172) for induction and maintenance. After induction with $5 \mathrm{~L} / \mathrm{min}$ oxygen and $8 \%$ sevoflurane through the mask, the anesthetists chose and inserted the modified first-generation singlelumen laryngeal mask airway of the appropriate model. Anesthetists adjusted sevoflurane concentration during maintenance according to vital signs and Bispectral Index (the majority between 40 and 60 for the suitable depth of anesthesia), mostly $2.5 \%$ to $3 \%$ with a $2 \mathrm{~L} / \mathrm{min}$ mixture of air and oxygen. In the whole process, the patients retained spontaneous breathing, and $\mathrm{PetCO}_{2}$ was usually maintained within 35 to $45 \mathrm{mmHg}$. Supplemental opioids, benzodiazepines, and other general anesthetics were not allowed, but local anesthetics were permitted to provide analgesia with the same dose as the local anesthesia group. There was postanesthesia care for $2 \mathrm{~h}$ after surgery. They were allowed to leave the hospital after the modified Aldrete score reached 10 points.

\section{Data collection and measure}

Before the operation, demographic data, pregnancy and birth details, and family structure were collected. During surgery, anesthetists recorded vital signs and perioperative adverse events. The patients were followed up by telephone on the day of surgery and the first day after surgery.

At six months after surgery, the WPPSI-IV (CN) was used to assess the neurodevelopment for two groups of children. It was to be done within one month after half a year after surgery. All children individually completed one-to-one with the qualified assessor. It took approximately $1.5 \mathrm{~h}$ for each child to complete the assessment. Parents were asked if children had been found physical abnormalities since caries treatment, and brief physical and neurological examinations were done for patients.

\section{Statistical analysis}

The hypothesis was that the FSIQ score is equivalent in children who have received general anesthesia or local anesthesia for caries treatment at six months after surgery. The equivalence margin was defined as five (1/3 SD), and a two one-sided test was performed with the 0.025 level of significance. Equivalence was predefined as the $95 \% \mathrm{Cl}$ of difference between the mean of the FSIQ score within -5 to +5 . The 
secondary outcomes, $\mathrm{VCl}, \mathrm{VSI}, \mathrm{FRI}, \mathrm{WMI}$, and PSI, also have a mean of 100 and a standard deviation of 15. It is reasonable to assume that their equivalence margins are five. All Cls are two-sided.

The statistical analysis plan prespecified the age at evaluation ( $<4$ years old or $\geq 4$ years old) as a subgroup analysis. Several predictor variables of primary outcome used in the regression model included anesthesia group, sex, gestational age at birth, birthweight, past medical histories of children, mother's abnormalities during pregnancy, mother's education, maternal age at delivery, perioperative adverse events, age at evaluation, and postoperative developmental disorders. In the general anesthesia group, the duration of sevoflurane exposure was used as a predictor variable and grouped (less than 120 min, 120 to $180 \mathrm{~min}$, and more than $180 \mathrm{~min}$ ) to observe the effect of different anesthesia duration on the primary outcome. All analyses were done in SPSS (version 25).

\section{Results}

\section{Trial process}

Between Feb 1, 2019, and Aug 31, 2019, 1681 children were screened for eligibility, and 340 patients were recruited. After three withdrawal of consent by parents (pre-surgery), the intent-to-treat analysis included 168 children in the general anesthesia group and 169 children in the local anesthesia group. 31 participants with surgery being canceled or protocol violations were excluded, and 150 patients in the general anesthesia completed the surgery and 156 in the local anesthesia group.

Follow-up was from Aug 1, 2019, to Mar 22, 2020. 28 families were lost to follow-up or withdrew consent, and one child was injured by fall from height. In the per-protocol analysis, the WPPSI-IV (CN) was complete for 129 in the general anesthesia group and 144 in the local anesthesia group (figure).

\section{Demographic data at baseline and non-scale measurement after surgery}

Demographic data at baseline and anesthesia details are shown in table 1 . There were 63 boys (49\%) in the general anesthesia group and 77 boys (53\%) in the local anesthesia group. The age at surgery was from 2.5 to 6.5 years old. Among all participants, 9 mothers had mild abnormalities during pregnancy, and 12 children had a past medical history. The median duration of sevoflurane use was $130 \mathrm{~min}$ (110 to 160). The only adverse event during the perioperative period in the general anesthesia group was related to respiratory complications (two cases of slight laryngospasm), and one case of the local anesthetic adverse reaction occurred in the local anesthesia group.

Non-scale measurement at six months after surgery are shown in table 2. There were 16 children less than 4 years old in the general anesthesia group and 9 children in the local anesthesia group. After surgery, no child had a diagnosis of febrile convulsion, epileptic seizure, or cerebral palsy, nor did they fail to complete WPPSI-IV (CN) due to developmental issues or behavioral disorders. The neurological examinations of all children were normal.

\section{Overall results of scale measurement}


The scores of FSIQ and main indexes (VCI, VSI, FRI, WMI, and PSI) in the WPPSI-IV (CN) of the two groups were shown in table 3. For the FSIQ score, the mean was 103.12 (SD 8.94) in the general anesthesia group and 103.58 (8.40) in the local anesthesia group. We noted evidence for equivalence between groups in means (mean difference for local anesthesia minus general anesthesia $0.46,95 \% \mathrm{Cl}-2.35$ to 1.61). There was also evidence for equivalence in the scores of $\mathrm{VCl}, \mathrm{VSI}, \mathrm{FRI}, \mathrm{WMI}$, and PSI between groups. The upper and lower limits of the $95 \%$ Cls were well inside the prespecified equivalence margin of five in all analyses.

\section{Subgroup analysis and predictor variables analysis of scale measurement}

In the subgroup analysis, the FSIQ score between groups was equivalent in the age group less than 4 years old. It was the same in the age group older than 4 years. There was no significant difference between the two age groups in the general anesthesia group and the local anesthesia group $(p>0.05)$. For the secondary outcomes, in the age subgroup of younger than 4 years, the VSI and WMI were not found to be equivalent between groups; in the age subgroup of older than 4 years, the VSI was not equivalent (table 4).

In the regression analysis, it was found that the anesthesia group, sex, preterm, birthweight, past medical histories of children, mother's pregnancy disease, maternal age at delivery, perioperative adverse events, and age at evaluation could not significantly affect the primary outcome $(p>0.05)$. Only the significance level of maternal education was less than $0.05(b=1.94$, R square $=1.9 \%)$, which could affect the FSIQ score (table 5). In the general anesthesia group, the duration of sevoflurane exposure did not significantly affect the primary outcome ( $p>0.05$ ), and there was no significant difference between different anesthesia durations ( $p>0.05)$.

Besides, we also analyzed the delivery mode and family structure and found that the cesarean section and second child could not significantly affect the primary outcome $(p>0.05)$.

\section{Discussion}

We noted evidence for equivalence between general anesthesia and local anesthesia in children for caries treatment in terms of the FSIQ in WPPSI-IV (CN) measured at six months after surgery. Equivalence was also shown in the $\mathrm{VCl}, \mathrm{VSI}, \mathrm{FRI}, \mathrm{WMI}$, and PSI, because the $95 \%$ Cls of the differences in means fell inside a third of an SD, well within our predefined margins of clinical equivalence. Local infiltration anesthesia in children is known to be safe and effective ${ }^{16}$. Therefore, it can be reasonably considered that children receiving prolonged DGA with sevoflurane alone had no adverse changes in neurocognitive development at six months after surgery.

The WPPSIIIV (CN) is a well-proven method for evaluating development, and the FSIQ score as the overall result is an appropriate evaluation indicator. Intelligence that covers multiple cognitive areas (memory, reasoning, spatial ability, executive ability, and processing speed) is deemed to be a relatively steady and constant trait of young children, and intellectual development in early childhood can predict 
and evaluate the future intelligence ${ }^{17}$. Consequently, we use the WPPSI-IV (CN), a validated, internationally recognized, and standardized measurement tool to assess the neurocognitive development and general intellectual of children. It has neuropsychological evaluation characteristics and can provide composite scores representing the intellectual function of specified cognitive domains (verbal IQ, performance IQ, and processing velocity) and an overall score for FSIQ ${ }^{18}$. There are previous studies assessing children exposed to anesthesia with medical and school records ${ }^{19,20}$. However, individually measured cognitive abilities are more sensitive than the performance of group-tested academic skills ${ }^{21}$.

From 3rd trimester to 24 months of age is the peak synaptogenesis of neurons, which is the most vulnerable to potential toxins. So many studies are directed at infants and children under 2 to 3 years old. However, brain development in children is dynamic and on-going. And infants undergoing surgery at a very young age are more likely to have underlying diseases, which may confuse the effects of general anesthesia exposure on neurodevelopmental outcomes. ${ }^{17}$ Therefore, we selected preschool children as the research object to observe whether the neurodevelopment in children after the peak synaptogenesis of neurons is susceptible to general anesthesia.

The combination of disease and surgery made previous retrospective studies challenging assess the independent effect of anesthesia exposure 20,22 . In the PANDA study, more than one anesthetic was used $^{13}$. The GAS trial was dominated by male infants (over $80 \%$ ), and the duration of surgery was less than $1 \mathrm{~h}$ (median $54 \mathrm{~min})^{10,11}$.

This trial only included healthy children who had no potential factors or disease that influenced neurodevelopment. For the surgery, just caries treatment with little painful noxious stimulation, and almost no effect on the body's internal environment was selected. The general anesthesia group used a sevoflurane-only anesthetic. It was to reduce the interference of the confounding factors of the disease, surgery, and the drug interaction of multiple general anesthetics. Considering that behavioral differences after general anesthesia in animal models may be owing to sex differences ${ }^{23}$, the ratio of male and female recruited was approximately 1:1. The duration of general anesthesia was in the range of 1 to $4 \mathrm{~h}$. The aim is to assess the effects of prolonged (non-transient) general anesthesia exposure on children (not just boys).

This is not a completely randomized trial. The choice of anesthesia method is determined by the cooperation of children and the parents. Among children with more severe dental caries and able to complete the dental procedure under local anesthesia, there are fewer children in younger age group than in the general anesthesia group. Therefore, there is a slight difference in the comparison between the two groups of children in height and weight (table 1 and table 2).

At present, there is no direct correlation between anesthesia and neurotoxicity of cognitive or behavioral sequelae in children ${ }^{24}$. Recent findings suggest that the anesthetic neurotoxicity is not a major contributor to adverse neurodevelopmental outcomes for most healthy children who require surgery. 
Biological, environmental, and social factors are far greater import ${ }^{25}$. In the subgroup analysis, we found that although the VSI and WMI scores in the secondary outcomes were not equivalent between groups. However, in the age subgroup of younger than 4 years, the average VSI score of the local anesthesia group minus the general anesthesia group was -5.45 , while the average WMI score of the local anesthesia group minus the general anesthesia group was 1.03 (table 4). That is, it is not that the general anesthesia group's score is always lower than that of the local anesthesia group. Therefore, this difference may be caused by environmental or other factors, rather than general anesthesia. We also found that the mother's education had an impact on the neurodevelopmental outcome in children. The higher the mother's education, the better comprehensive neurocognitive ability on children, but the influence is not great.

Inconsistent with other research conclusions ${ }^{14,23}$, we did not find that premature, the duration of sevoflurane exposure, and the age of children had a significant effect on neurocognitive outcome. It was similar in maternal abnormalities during pregnancy and past medical histories of children. Because after the screening, moderate to severe preterm infants were excluded, and these participants just had the mild disease in the past. They were relatively healthy.

Awake-local anesthesia inevitably has a failure rate. It was unavoidable that children may refuse surgery or change to general anesthesia due to dental anxiety. If all children were analyzed on an intention-totreat basis, the results obtained would be more conservative and dilute the potential effect of general anesthesia, making the differences between groups more difficult to detect. To minimize this bias and ensure the reliability of the results, we analyzed mainly on a per-protocol basis.

Our trial has several limitations. The local anesthesia group could not compare the duration of surgery and anesthesia with the general anesthesia group. Although the two groups of children have similar severity of caries, almost all children with multiple caries could not complete all treatments at once with local anesthesia. It was usually required multiple visits so that the duration could not be counted. Therefore, the advantage of DGA is to provide convenient and efficient treatment. The general anesthesia group used a sevoflurane-only anesthetic without other general anesthetics. Thus, our trial cannot be generalized to practice in all pediatric surgery, and cannot elucidate possible outcomes if other agents are added.

Another limitation is that the children were recruited mainly in Chongqing, Southwest China. The findings may be influenced by the differences from region and economy. At present we have only evaluated the participants at six months after surgery. To avoid the practice effect, after the recommended interval (one year), our research plan is to perform the subsequent evaluation of participants, in order to more accurately describe long-term neurocognitive outcomes after DGA.

\section{Conclusion}

This trial, being congruent with data from several previous clinical studies, found no evidence that prolonged exposure to DGA only with sevoflurane in relatively healthy preschool children causes adverse 
neurodevelopmental outcomes (including language, reasoning, memory, and vision-action) and neurological deficits at six months after surgery. These findings should reassure pediatricians and parents considering delaying surgery due to developmental disorders and medical risks. The longer-term neurodevelopmental outcomes after anesthesia exposure need to be assessed in the future.

\section{Abbreviations}

DGA (dental procedures under general anesthesia), WPPSI (CN) (The Wechsler Preschool and Primary Scale of Intelligence-Fourth Edition Chinese version), FSIQ (the Full-Scale IQ), VCI (Verbal Comprehension Index), VSI (Visual-Spatial Index), FRI (Fluid Reasoning Index), WMI (Working Memory Index), PSI (Processing Speed Index).

\section{Declarations}

\section{Ethics approval and consent to participate}

The study had been treated according to the declaration of Helsinki. This trial was registered with Chinese Clinical Trial Registry (number ChiCTR1800015216), and the ethics committee approval of the Stomatological Hospital of Chongqing Medical University was obtained (number CQHS-IRB-2018-01). The written consent of all participants obtained from parents or guardians.

\section{Consent to publish}

All authors read the final manuscript and agreed with submission to BMC Anesthesiology.

\section{Availability of data and materials}

The datasets used and/or analysed during the current study are available from the corresponding author on reasonable request.

\section{Competing interests}

The authors declare that they have no competing interests in this trial.

\section{Funding}

This work was supported by the Research Project of Chongqing Health Bureau (grant number 2017ZDXM017). The funders had no role in study design, data collection and analysis, decision to publish, or preparation of the manuscript.

\section{Authors' Contributions}

Study conception and design: CY, GJH, PPZ 
Data acquisition: WZM, YH, CZ, PPZ

Analysis and data interpretation: CZ, PPZ

Drafting of the manuscript: PPZ

Critical revision: $\mathrm{CY}, \mathrm{CZ}$, GJH

\section{Acknowledgments}

We would like to thank all children and guardians participating in the trial, and the anesthetists and pediatric dentists of the Stomatological Hospital of Chongqing Medical University.

\section{Authors' Information}

Professor Cong Yu is the director of the Department of Anesthesiology of the Stomatological Hospital of Chongqing Medical University. The Stomatological Hospital of Chongqing Medical University started using general anesthesia for ambulatory dental treatment in children since 2010, and it is the first hospital starting this technique in Southwest China. The number of general anesthesia for dental treatment exceeds 1,400 cases per year, which provides a basis for verifying the effect of this technique on the neurodevelopment of children.

\section{References}

1. Du MQ, Li Z, Jiang H, et al. Dental Caries Status and its Associated Factors among 3- to 5-year-old Children in China: A National Survey. Chin J Dent Res. 2018;21(3):167-179.

2. Blumer S, Costa L, Peretz B. Success of Dental Treatments under Behavior Management, Sedation and General Anesthesia. J Clin Pediatr Dent. 2017;41(4):308-311.

3. Blumer S, Costa L, Peretz B. Success of Dental Treatments under Behavior Management, Sedation and General Anesthesia. J Clin Pediatr Dent. 2019;43(6):413-416.

4. Chao Z, Gui Jin H, Cong Y. The effect of general anesthesia for ambulatory dental treatment on children in Chongqing, Southwest China. Paediatric anaesthesia. 2017;27(1):98-105.

5. Briner A, Nikonenko I, De Roo M, Dayer A, Muller D, Vutskits L. Developmental Stage-dependent persistent impact of propofol anesthesia on dendritic spines in the rat medial prefrontal cortex. Anesthesiology. 2011;115(2):282-293.

6. Paule MG, Li M, Allen RR, et al. Ketamine anesthesia during the first week of life can cause longlasting cognitive deficits in rhesus monkeys. Neurotoxicol Teratol. 2011;33(2):220-230.

7. Schaefer ML, Wang M, Perez PJ, Coca Peralta W, Xu J, Johns RA. Nitric Oxide Donor Prevents Neonatal Isoflurane-induced Impairments in Synaptic Plasticity and Memory. Anesthesiology. 2019;130(2):247-262. 
8. Davidson AJ, Sun LS. Clinical Evidence for Any Effect of Anesthesia on the Developing Brain. Anesthesiology. 2018;128(4):840-853.

9. Andropoulos DB. Effect of Anesthesia on the Developing Brain: Infant and Fetus. Fetal Diagn Ther. 2018;43(1):1-11.

10. Davidson AJ, Disma N, de Graaff JC, et al. Neurodevelopmental outcome at 2 years of age after general anaesthesia and awake-regional anaesthesia in infancy (GAS): an international multicentre, randomised controlled trial. Lancet. 2016;387(10015):239-250.

11. McCann ME, de Graaff JC, Dorris L, et al. Neurodevelopmental outcome at 5 years of age after general anaesthesia or awake-regional anaesthesia in infancy (GAS): an international, multicentre, randomised, controlled equivalence trial. Lancet. 2019;393(10172):664-677.

12. Glatz P, Sandin RH, Pedersen NL, Bonamy AK, Eriksson LI, Granath F. Association of Anesthesia and Surgery During Childhood With Long-term Academic Performance. JAMA Pediatr. 2017;171(1):e163470.

13. Sun LS, Li G, Miller TL, et al. Association Between a Single General Anesthesia Exposure Before Age 36 Months and Neurocognitive Outcomes in Later Childhood. Jama. 2016;315(21):2312-2320.

14. Zhang Q, Peng $Y$, Wang Y. Long-duration general anesthesia influences the intelligence of school age children. BMC Anesthesiol. 2017;17(1):170.

15. Oba S, Türk H. A case report of multiple anesthesia for pediatric surgery: 80 anesthesia applications in a period of 6 years. BMC Anesthesiol. 2018;18(1):175.

16. Gunter JB. Benefit and risks of local anesthetics in infants and children. Paediatr Drugs. 2002;4(10):649-672.

17. Clausen NG, Kahler S, Hansen TG. Systematic review of the neurocognitive outcomes used in studies of paediatric anaesthesia neurotoxicity. Br J Anaesth. 2018;120(6):1255-1273.

18. McNulty $H$, Rollins $M$, Cassidy $T$, et al. Effect of continued folic acid supplementation beyond the first trimester of pregnancy on cognitive performance in the child: a follow-up study from a randomized controlled trial (FASSTT Offspring Trial). BMC Med. 2019;17(1):196.

19. Schneuer FJ, Bentley JP, Davidson AJ, et al. The impact of general anesthesia on child development and school performance: a population-based study. Paediatr Anaesth. 2018;28(6):528-536.

20. Hu D, Flick RP, Zaccariello MJ, et al. Association between Exposure of Young Children to Procedures Requiring General Anesthesia and Learning and Behavioral Outcomes in a Population-based Birth Cohort. Anesthesiology. 2017;127(2):227-240.

21. Lin EP, Lee JR, Lee CS, Deng M, Loepke AW. Do anesthetics harm the developing human brain? An integrative analysis of animal and human studies. Neurotoxicol Teratol. 2017;60:117-128.

22. Warner DO, Zaccariello MJ, Katusic SK, et al. Neuropsychological and Behavioral Outcomes after Exposure of Young Children to Procedures Requiring General Anesthesia: The Mayo Anesthesia Safety in Kids (MASK) Study. Anesthesiology. 2018;129(1):89-105. 
23. Vutskits L, Davidson A. Update on developmental anesthesia neurotoxicity. Curr Opin Anaesthesiol. 2017;30(3):337-342.

24. Earley MA, Pham LT, April MM. Scoping review: Awareness of neurotoxicity from anesthesia in children in otolaryngology literature. Laryngoscope. 2017;127(8):1930-1937.

25. Graham MR. Clinical update regarding general anesthesia-associated neurotoxicity in infants and children. Curr Opin Anaesthesiol. 2017;30(6):682-687.

\section{Tables}

Table 1: Demographic data at baseline and anesthesia details 
GA group ( $n=129) \quad$ LA group $(n=144)$

vaule*

Baseline demographics

Sex, M/F

$63 / 66$

$77 / 67$

0.444

Age at surgery

$<4$ years old

36

22

0.011

$\geq 4$ years old

93

122

Weight, kg; median (IQR)

Height, cm; median (IQR)

$\begin{array}{ccc}17.50(15.80-18.70) & 18.35(16.50-20.20) & <0.001 \\ 106.00(99.50- & 106.00(101.00- & 0.003 \\ 110.00) & 115.00) & \\ & & \end{array}$

Past medical history

Non-recurrent febrile convulsion

$2(2 \%)$

$3(2 \%)$

1.000

Thalassemia without a history of blood

$3(2 \%)$

0.104 transfusion

Favism

Patent ductus arteriosus without treatment

0

Pregnancy and birth details

Preterm (born <37 weeks' gestation)

$2(2 \%)$

$1(\nabla 1 \%)$

1.000

Birthweight, kg

$8(6 \%)$

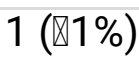

0.604

One of a multiple pregnancy

$3.30(0.53)$

$5(3 \%)$

0.290

$2(2 \%)$

$3.29(0.46)$

0.909

Abnormal pregnancy examination of mother

$4(3 \%)$

$1(\mathbb{1} \%)$

0.604

Delivery mode

Cephalic vaginal

$62(48 \%)$

$5(3 \%)$

1.000

Cesarean section with intravertebral anesthesia

Family demographics

Maternal age at birth more than 30 years

$67(52 \%)$

$73(51 \%)$

0.664

Maternal education

Junior high school

Senior high school

$33(26 \%)$

$71(49 \%)$

Bachelor degree

Master degree or higher

Number of children in the family

1

2

3

Birth order

1
2
3

\section{Anaesthesia details}

Duration of surgery, min; median (IQR)

$7(5 \%)$

$31(24 \%)$

$82(64 \%)$

$9(7 \%)$

$83(64 \%)$

$45(35 \%)$

$1(\mathbb{1} \%)$

$108(84 \%)$

$21(16 \%)$

0
$45(31 \%)$

$5(3 \%)$

$27(19 \%)$

$108(75 \%)$

$4(3 \%)$

$92(64 \%)$

0.909

$50(35 \%)$

$2(1 \%)$

$114(79 \%)$

$29(20 \%)$

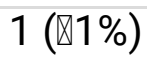

Duration of use of sevoflurane, min; median (IQR)

Adverse events related to the cardiovascular system

Adverse events related to the respiratory system
$115(95-140)$
$130(110-160)$

0

NA

NA

$1(\mathbb{\nabla} \%) \dagger$

1.000

$2(2 \%) \ddagger$

0.222 
Data are presented as $\mathrm{n}$, means (standard deviations), or median (IQR). GA=general anesthesia. $\mathrm{LA}=$ local anesthesia.

*Pearson's chi-squared test, Fisher's exact test, Rank sum test, or Two independent samples t-test.

tOne patient had an adverse reaction of local anesthetic with increased heart rate (recovered after treatment).

$\ddagger$ Two children had mild laryngospasm without evident hypoxia during anesthesia recovery.

$\S$ Apparent hypoxia defined as oxygen saturation $<90 \%$.

Table 2: Non-scale measurement at six months after surgery

\begin{tabular}{|c|c|c|c|}
\hline & GA group $(n=129)$ & LA group $(n=144)$ & $\begin{array}{c}P \\
\text { vaule }\end{array}$ \\
\hline \multicolumn{4}{|l|}{ Assessment details } \\
\hline \multicolumn{4}{|l|}{ Age at follow-up assessment } \\
\hline$<4$ years old & 16 & 9 & 0.078 \\
\hline$\geq 4$ years old & 113 & 135 & \\
\hline Weight, kg; median (IQR) & $19.60(17.90-20.85)$ & $20.20(18.60-22.10)$ & 0.003 \\
\hline Height, cm; median (IQR) & $\begin{array}{l}110.00(103.00- \\
114.00)\end{array}$ & $\begin{array}{l}110.00(106.00- \\
119.00)\end{array}$ & 0.005 \\
\hline Abnormal neurological examinations & 0 & 0 & \\
\hline \multicolumn{4}{|l|}{ Events after caries treatment } \\
\hline Febrile convulsion or epileptic seizure & 0 & 0 & \\
\hline Cerebral palsy & 0 & 0 & \\
\hline Developmental delay & 0 & 0 & \\
\hline Hearing or vision impairment & 0 & 0 & \\
\hline $\begin{array}{l}\text { Language, behavior or psychomotor } \\
\text { disorder }\end{array}$ & 0 & 0 & \\
\hline $\begin{array}{l}\text { Intervention for neurodevelopmental } \\
\text { problem }\end{array}$ & 0 & 0 & \\
\hline
\end{tabular}

Table3: Comparison of WPPSI- $(\mathrm{CN})$ scores between groups 


\begin{tabular}{lcccc}
\hline $\begin{array}{c}\text { Composite } \\
\text { score }\end{array}$ & $\begin{array}{c}\text { GA group } \\
(\mathbf{n = 1 2 9 )}\end{array}$ & $\begin{array}{c}\text { LA group } \\
(\mathbf{n = 1 4 4 )}\end{array}$ & $\begin{array}{c}\text { Difference } \\
\text { in LA-GA }\end{array}$ & $\begin{array}{c}\text { 95\% CI for difference } \\
\text { in LA-GA }\end{array}$ \\
\hline FSIQ & $103.12(8.94)$ & $103.58(8.40)$ & 0.46 & -2.53 to 1.61 \\
\hline VCI & $105.57(10.72)$ & $104.81(13.61)$ & -0.76 & -2.18 to 3.70 \\
\hline VSI & $102.12(13.30)$ & $104.09(10.73)$ & 1.97 & -4.84 to 0.90 \\
\hline FRI & $105.44(9.89) \dagger$ & $106.07(12.33) \neq$ & 0.63 & -3.46 to 2.20 \\
\hline WMI & $101.10(11.28)$ & $101.64(11.58)$ & 0.54 & -3.27 to 2.19 \\
\hline PSI & $106.71(9.96) \dagger$ & $105.46(11.02) \neq$ & -1.25 & -1.40 to 3.90 \\
\hline
\end{tabular}

Data are means (SD). GA=general anesthesia. LA=local anesthesia. WPPSI- $-(\mathrm{CN})$ $=$ Wechsler Preschool and Primary Scale of Intelligence-Fourth Edition (Chinese version).FSIQ=Full Scale IQ. VCI=Verbal Comprehension Index. VSI=Visual Spatial Index. FRI=Fluid Reasoning Index. WMI=Working Memory Index. PSI=Processing Speed Index. †There is no FRI and PSI in the age group of less than 4 years $(n=113)$. ‡There is no FRI and PSI in the age group of less than 4 years $(n=135)$.

Table4: Comparison of WPPSI(CN) scores in age subgroups

\begin{tabular}{|c|c|c|c|c|c|c|}
\hline $\begin{array}{l}\text { Composite } \\
\text { score }\end{array}$ & $\begin{array}{l}<4 \text { years } \\
\text { old } \\
\text { in GA } \\
\text { group } \\
(\mathrm{n}=16)\end{array}$ & $\begin{array}{l}<4 \text { years } \\
\text { old } \\
\text { in LA } \\
\text { group } \\
(\mathrm{n}=9)\end{array}$ & $\begin{array}{l}\text { Difference }(< \\
4 \text { years old) } \\
\text { in LA-GA }(95 \% \\
\text { CI) }\end{array}$ & $\begin{array}{l}\geq 4 \text { years } \\
\text { old } \\
\text { n GA group } \\
(\mathrm{n}=113)\end{array}$ & $\begin{array}{l}\geq 4 \text { years } \\
\text { old } \\
\text { in } \underset{(n=135)}{(n=1350}\end{array}$ & $\begin{array}{l}\text { Difference }(\geq 4 \\
\text { years old) } \\
\text { in LA-GA }(95 \% \\
\text { CI) }\end{array}$ \\
\hline$\overline{S I Q}$ & $\begin{array}{l}101.56 \\
(4.72)\end{array}$ & $\begin{array}{l}102.33 \\
(5.20)\end{array}$ & $\begin{array}{c}0.77(-4.99 \text { to } \\
3.45)\end{array}$ & $\begin{array}{l}103.58 \\
(9.17)\end{array}$ & $\begin{array}{l}103.67 \\
(8.58)\end{array}$ & $\begin{array}{r}0.09 \\
2 .\end{array}$ \\
\hline VCI & $\begin{array}{l}103.69 \\
(5.49)\end{array}$ & $\begin{array}{l}103.78 \\
(5.14)\end{array}$ & $\begin{array}{c}0.09(-4.72 \text { to } \\
4.54)\end{array}$ & $\begin{array}{l}105.26 \\
(11.05)\end{array}$ & $\begin{array}{l}104.70 \\
(13.65)\end{array}$ & .59 to \\
\hline VSI & $\begin{array}{l}103.56 \\
(9.14)\end{array}$ & $\begin{array}{l}98.11 \\
(7.13)\end{array}$ & $\begin{array}{r}-5.45(- \\
12.7\end{array}$ & $\begin{array}{l}101.86 \\
(13.64)\end{array}$ & $\begin{array}{l}104.49 \\
(10.83)\end{array}$ & $\begin{array}{l}69 \text { to } \\
) \neq\end{array}$ \\
\hline FRI & NA $†$ & $\mathrm{NA} \dagger$ & & $\begin{array}{l}105.44 \\
(9.89)\end{array}$ & $\begin{array}{l}106.07 \\
(12.33)\end{array}$ & $\begin{array}{r}0.63( \\
2 .\end{array}$ \\
\hline WMI & $\begin{array}{l}104.75 \\
(7.00)\end{array}$ & $\begin{array}{l}105.78 \\
(6.16)\end{array}$ & $\begin{array}{r}1.03(-6 \\
4.76\end{array}$ & & $\begin{array}{l}101.67 \\
(11.64)\end{array}$ & $\begin{array}{c}1.07(-3.94 \text { to } \\
1.80)\end{array}$ \\
\hline SI & NA $†$ & NA $†$ & & $\begin{array}{l}106.71 \\
(9.96)\end{array}$ & $\begin{array}{l}105.46 \\
(11.02)\end{array}$ & $\begin{array}{c}-1.25(-1.40 \text { to } \\
3.90)\end{array}$ \\
\hline \multicolumn{7}{|c|}{$\begin{array}{l}\text { Data are means (SD). GA=general anesthesia. LA=local anesthesia. WPPSI- }(\mathrm{CN}) \\
\text { =Wechsler Preschool and Primary Scale of Intelligence-Fourth Edition (Chinese version). } \\
\text { FSIQ=Full Scale IQ. VCI=Verbal Comprehension Index. VSI=Visual Spatial Index. } \\
\text { FRI=Fluid Reasoning Index. WMI=Working Memory Index. PSI=Processing Speed Index. } \\
\text { tThere is no FRI and PSI in the age group of less than } 4 \text { years. } \neq \text { There is no equivalence. }\end{array}$} \\
\hline
\end{tabular}


Table 5: Regression analysis of predictor variables

\begin{tabular}{lccc}
\hline \multicolumn{1}{c}{ predictor variables } & P vaule & b & R square \\
\hline anesthesia group & 0.787 & & \\
\hline sex & 0.263 & & \\
\hline preterm & 0.694 & & \\
birthweight & 0.977 & & \\
\hline past medical histories of children & 0.715 & & \\
mother's pregnancy disease & 0.218 & & \\
mother's education & $0.022^{*}$ & 1.941 & $1.90 \%$ \\
\hline maternal age at delivery & 0.192 & & \\
perioperative adverse events & 0.916 & & \\
age at evaluation & 0.476 & & \\
* The significance level< 0.05. & & & \\
\hline
\end{tabular}

\section{Figures}

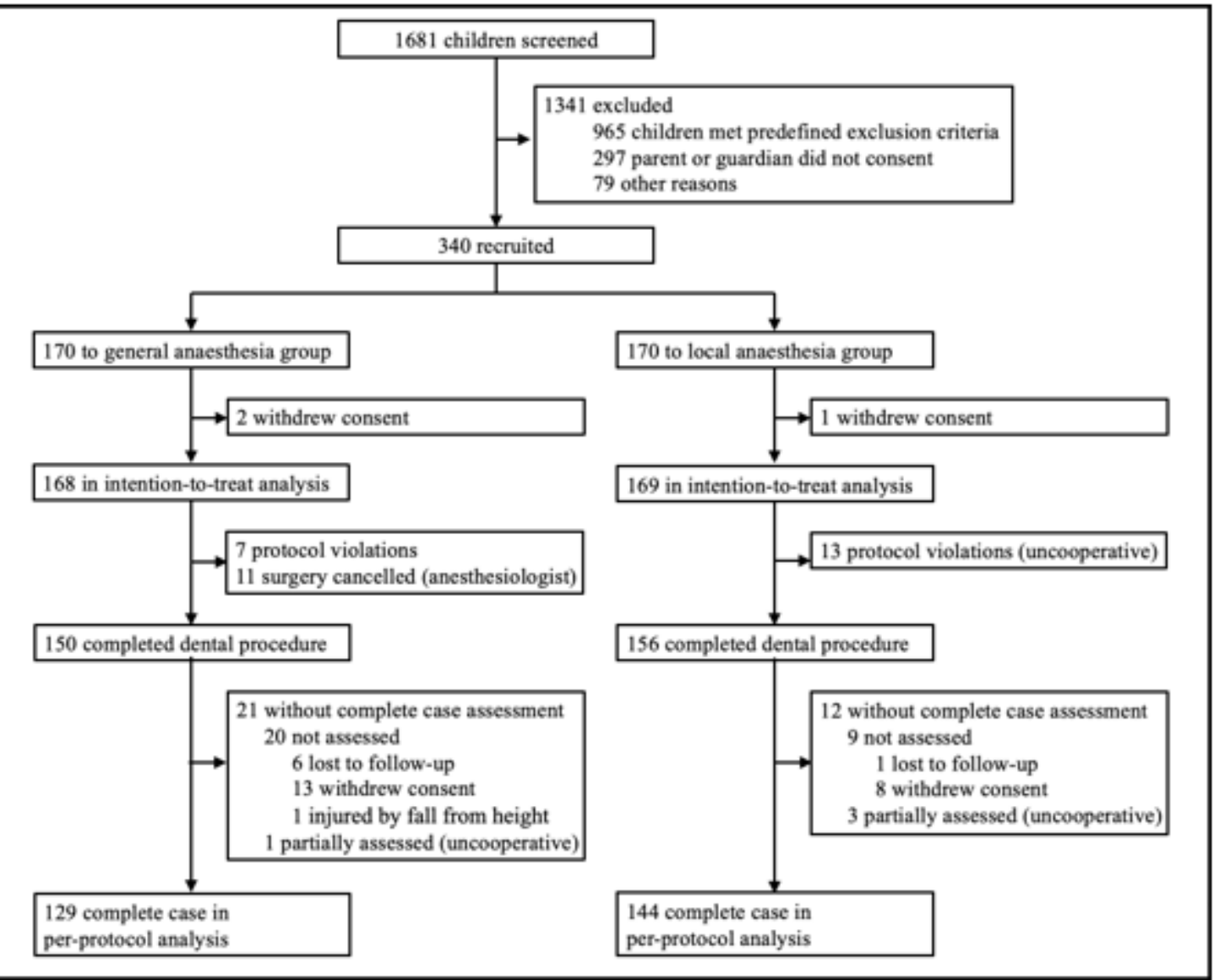


Figure 1

Trial profile

Page $17 / 17$ 